\title{
High-resolution intracranial vessel wall MRI in an elderly asymptomatic population: comparison of 3T and 7T
}

\author{
Anita A. Harteveld ${ }^{1}$ • Anja G. van der Kolk ${ }^{1}$ H. Bart van der Worp ${ }^{2} \cdot$ Nikki Dieleman $^{1}$ • \\ Jeroen C. W. Siero ${ }^{1}$ - Hugo J. Kuijf ${ }^{3}$ - Catharina J. M. Frijns ${ }^{2} \cdot$ Peter R. Luijten $^{1}$ • \\ Jaco J. M. Zwanenburg ${ }^{1}$. Jeroen Hendrikse ${ }^{1}$
}

Received: 2 March 2016 /Revised: 7 June 2016 / Accepted: 21 June 2016 / Published online: 7 July 2016

(C) The Author(s) 2016. This article is published with open access at Springerlink.com

\begin{abstract}
Objectives Several intracranial vessel wall sequences have been described in recent literature, with either 3-T or 7-T magnetic resonance imaging (MRI). In the current study, we compared 3-T and 7-T MRI in visualising both the intracranial arterial vessel wall and vessel wall lesions.

Methods Twenty-one elderly asymptomatic volunteers were scanned by 3-T and 7-T MRI with an intracranial vessel wall sequence, both before and after contrast administration. Two raters scored image quality, and presence and characteristics of vessel wall lesions.

Results Vessel wall visibility was equal or significantly better at $7 \mathrm{~T}$ for the studied arterial segments, even though there were more artefacts hampering assessment. The better visualisation of the vessel wall at $7 \mathrm{~T}$ was most prominent in the proximal anterior cerebral circulation and the posterior cerebral artery. In the studied elderly asymptomatic population, 48 vesselwall lesions were identified at $3 \mathrm{~T}$, of which 7 showed enhancement. At 7 T, 79 lesions were identified, of which 29 showed enhancement. Seventy-one percent of all 3-T lesions
\end{abstract}

Electronic supplementary material The online version of this article (doi:10.1007/s00330-016-4483-3) contains supplementary material, which is available to authorized users.

Anita A. Harteveld

a.a.harteveld-2@umcutrecht.nl

1 Department of Radiology, University Medical Center Utrecht, Postbox 85500, 3508 GA Utrecht, The Netherlands

2 Department of Neurology and Neurosurgery, Brain Center Rudolf Magnus, University Medical Center Utrecht,

Utrecht, The Netherlands

3 Image Sciences Institute, University Medical Center Utrecht, Utrecht, The Netherlands and $59 \%$ of all 7-T lesions were also seen at the other field strength.

Conclusions Despite the large variability in detected lesions at both field strengths, we believe 7-T MRI has the highest potential to identify the total burden of intracranial vessel wall lesions.

Key Points

- Intracranial vessel wall visibility was equal or significantly better at 7-T MRI

- Most vessel wall lesions in the cerebral arteries were found at 7-T MRI

- Many intracranial vessel wall lesions showed enhancement after contrast administration

- Large variability in detected intracranial vessel wall lesions at both field strengths

- Seven-tesla MRI has the highest potential to identify total burden of intracranial atherosclerosis

Keywords Intracranial atherosclerosis $\cdot$ Magnetic resonance imaging $\cdot$ Cerebral arteries $\cdot$ Cerebrovascular disorders . Neuroimaging

\section{Introduction}

Intracranial atherosclerosis is one of the main causes of ischaemic stroke and transient ischaemic attack (TIA), and has been associated with a higher risk of recurrent stroke $[1,2]$. Current standard non-invasive imaging methods, like computed tomography (CT) angiography, visualise the lumen of the intracranial vasculature instead of its vessel wall, thereby risking underestimation of intracranial atherosclerosis in ischaemic stroke [3-6] due to compensatory arterial remodelling that maintains lumen diameter [4, 7]. Therefore, several magnetic resonance imaging (MRI) sequences have recently been 
developed for direct evaluation of the intracranial vessel wall and its pathology in vivo [8]. Studies have shown that highresolution MRI is able to identify intracranial vessel wall abnormalities even before causing luminal narrowing.

Intracranial vessel wall imaging has been performed mainly at 3.0- and 7.0-tesla (T) field strengths. At these higher magnetic field strengths, the increased signal-to-noise (SNR) and contrast-to-noise ratios (CNR) can be exploited for imaging at a higher spatial resolution within reasonable scan times to clearly show the thin arterial vessel walls, and for improvement of lesion conspicuousness [7, 9]. Current challenges at 3 $\mathrm{T}$ are incomplete cerebrospinal fluid (CSF) suppression, potentially limiting vessel wall assessment, and (often) limited coverage $[8,10,11]$. Seven-tesla MRI offers the advantage of an increased SNR, allowing for complete CSF suppression and whole-brain imaging within clinically feasible scan times [12]; its use, however, is currently hampered by restricted availability and increased transmit field $\left(\mathrm{B}_{1}{ }^{+}\right)$inhomogeneity, causing artefacts that can limit vessel wall assessment [13]. The implications of these differences are unclear, because a direct comparison between intracranial vessel wall MRI at these field strengths has not yet been performed. The purpose of this study was to compare visualisation of the intracranial vessel wall and possible vessel wall lesions between 3-T and 7-T MRI.

\section{Methods}

\section{Study population}

This prospective study was approved by the institutional review board of our institution; all subjects provided written informed consent. Between November 2013 and December 2014 , volunteers aged $>50$ years, without a history of cerebrovascular or ischaemic heart disease or contraindications for MR imaging, were included. These volunteers formed the control group of the ongoing PIVI study (Posterior Intracranial Vessel wall Imaging; NTR5688, www.trialregister.nl).

\section{Imaging}

All subjects underwent MRI at both $3 \mathrm{~T}$ and $7 \mathrm{~T}$. For each field strength, an optimised, $\mathrm{T}_{1}$-weighted intracranial vessel wall imaging sequence was used; a previous ex vivo study at $7 \mathrm{~T}$ [14] showed $\mathrm{T}_{1}$-weighted imaging to have the most promising image contrast for visualising and characterising intracranial arterial vessel wall lesions. In addition, a $\mathrm{T}_{1}$-weighted sequence enables assessment of vessel-wall contrast enhancement. The sequence at $3 \mathrm{~T}$ had been optimised in volunteers (data not shown) based on a previously published sequence by Qiao et al. [10] The 7-T sequence had already been optimised in previous studies $[12,15]$. At both field strengths, the intracranial vessel wall scan was performed before and after contrast administration; a minimum of $12 \mathrm{~h}$ was taken between both MRI examinations, to make sure the contrast agent had washed out sufficiently, and care was taken that both MRI examinations were planned as close after each other as possible (median, 4 days; IQR, 3-9 days). The postcontrast vessel wall scan was acquired approximately $5 \mathrm{~min}$ after intravenous administration of $0.1 \mathrm{~mL} / \mathrm{kg}$ of a gadolinium-containing contrast agent (Gadobutrol, Gadovist $1.0 \mathrm{mmol} / \mathrm{mL}$; Bayer Schering Pharma, Newbury, UK). For signal improvement in the cerebellar region at $7 \mathrm{~T}$, high permittivity dielectric pads were used (Leiden University Medical Center, Leiden, The Netherlands) [16, 17].

\section{3-T MRI protocol}

Imaging was performed on a 3-T whole-body system (Achieva; Philips Healthcare, Best, The Netherlands), with a quadrature body coil for transmission and an eight-channel head coil for reception. The imaging protocol included a three-dimensional (3D) $\mathrm{T}_{1}$-weighted volumetric isotropically reconstructed turbo spin-echo acquisition (VIRTA) intracranial vessel wall sequence (adapted from Qiao et al. [10] by Dieleman et al., submitted for publication). The applied scan parameters are presented in Table 1.

\section{7-T MRI protocol}

For 7-T MRI, a whole-body system (Philips Healthcare, Cleveland, OH, USA) was used with a 32-channel receive coil and volume transmit/receive coil for transmission (Nova Medical, Wilmington, MA, USA). The imaging protocol included a $3 \mathrm{D}$ whole-brain $\mathrm{T}_{1}$-weighted magnetisation-prepared inversion recovery turbo spin echo (MPIR-TSE) intracranial vessel wall sequence [15]. The applied scan parameters are presented in Table 1.

\section{Image assessment}

Images were assessed in the same format as they are normally evaluated in clinical practice, which includes the 'standard' image interpolation performed by the scanner software. Multiplanar reconstructions (MPRs) were made from the vessel-wall scans acquired at $3 \mathrm{~T}$ (thickness, $1.0 \mathrm{~mm}$; no slice gap) and $7 \mathrm{~T}$ (thickness, $0.8 \mathrm{~mm}$; no slice gap), using a standalone workstation (Philips). The used slice thickness was based on the acquired resolution in the slice direction. To obtain the same data sets for further analysis, the 7-T vessel-wall MPRs were made using the spatial orientation of the 3-T images.

Three-tesla and 7-T MPR images were evaluated in random order by two trained raters (A.K. and A.H.), with 6 and 3 years of experience in assessing intracranial vessel wall images, respectively, who were blinded for each other's 
Table 1 Scan parameters of the intracranial vessel wall imaging sequence at $3 \mathrm{~T}$ and $7 \mathrm{~T}$

\begin{tabular}{lll}
\hline Scan parameter & 3-T VIRTA & 7-T MPIR-TSE \\
\hline FOV $\left(\mathrm{mm}^{3}\right)$ & $200 \times 167 \times 45$ & $250 \times 250 \times 190$ \\
Acquisition orientation & Transverse oblique & Sagittal \\
Acquired spatial resolution $\left(\mathrm{mm}^{3}\right)$ & $0.6 \times 0.6 \times 1.0$ & $0.8 \times 0.8 \times 0.8$ \\
Reconstructed spatial resolution $\left(\mathrm{mm}^{3}\right)$ & $0.5 \times 0.5 \times 0.5$ & $0.49 \times 0.49 \times 0.49$ \\
TR / TE / TI (ms) & $1,500 / 36 /-$ & $3,952 / 37 / 1,375$ \\
Flip angle (degrees) & 90 & 120 \\
Echo spacing (ms) & 4.0 & 3.3 \\
TSE factor & 62 (incl. 6 start-up) & 169 (incl. 10 start-up) \\
Oversampling factor & 1.8 & 1 \\
NSA & 1 & 2 \\
SENSE factor & 1.5 (RL) & 2 (AP) \& 3 (RL) \\
Acquisition time (min:s) & $6: 51$ & $10: 40$ \\
\hline
\end{tabular}

FOV field-of-view, MPIR-TSE magnetisation-prepared inversion recovery, NSA number of signal averages, SENSE sensitivity encoding, TE echo time, TI inversion time, TR repetition time, TSE turbo spin echo, VIRTA volumetric isotropically reconstructed turbo spin-echo acquisition

${ }^{a}$ Sequence was planned so that as much of the circle of Willis as possible was within the FOV. Several parameters were adapted/added to Qiao et al. [10]: TR $=1,500 \mathrm{~ms}$ and anti-DRIVen Equilibrium (DRIVE) module to increase $\mathrm{T}_{1}$-weighting and CSF suppression; a low minimum flip angle ( 25 degrees) in the variable flip angle refocusing pulse train for increased flow suppression [18]; interpolation factor $=2$ by zero-padding in the slice direction during reconstruction, slight adjustment of the acquired in-plane resolution, and reducing the TR to reduce scan time further

${ }^{\mathrm{b}}$ In comparison to previous studies [12, 15, 19], sequences were obtained with a dual transmit system that provides $\mathrm{a} \mathrm{B}_{1}{ }^{+}$in the brain that matches the nominal flip angle; therefore, all flip angles could be reduced by $20 \%$ to obtain the same image contrast assessment and for the findings on the other (3-T or 7-T) scan. Each scan was analysed once by each rater; in case of disagreement regarding vessel wall lesions, a consensus reading was performed with a third rater (J.H.; 6 years of experience in assessing intracranial vessel wall images). After consensus, non-corresponding vessel-wall lesions at $3 \mathrm{~T}$ and $7 \mathrm{~T}$ were assessed in a side-by-side comparison.

The assessed vessel segments included: anterior cerebral artery (ACA; $\mathrm{A} 1$ and $\mathrm{A} 2$ segments); anterior communicating artery (ACoA); middle cerebral artery (MCA; M1, M2 and M3 segments); internal carotid artery (ICA; distal intracranial segment; intracranial bifurcation); posterior communicating artery $(\mathrm{PCoA})$; posterior cerebral artery (PCA; P1 segment, bifurcation $\mathrm{P} 1-\mathrm{P} 2$ and $\mathrm{P} 2$ segment); basilar artery (BA; proximal half, distal half and bifurcation with P1 segment); vertebral arteries (VA; proximal and distal half).

\section{Image quality}

Image quality was evaluated using the method modified from Van der Kolk et al. [15], with three qualitative grading scales for overall artefacts $(0=$ artefacts hampering assessment; $1=$ moderate artefacts, but images assessable; $2=$ no artefacts), overall visibility of the arterial vessel wall $(0=$ poor; 1 = moderate; 2 =good), and visibility of all separate arterial vessel walls $(0=$ outside FOV; $1=$ not visible; $2=$ poor; $3=$ moderate; 4 = good).

\section{Vessel-wall lesions}

Vessel-wall lesions were scored on the precontrast images according to the methods previously described $[12,20] \mathrm{A}$ vessel-wall lesion was defined as either a clear focal or more diffuse thickening of the vessel wall, compared with the healthy contralateral or neighbouring vessel wall [21]. For assessment of contrast enhancement, vessel wall images were processed using MeVisLab (version 2.7; MeVis Medical Solutions, Bremen, Germany). After coregistering the postcontrast scan to the precontrast scan using elastix [22], the precontrast scan was subtracted from the (coregistered) postcontrast scan. The resulting subtracted images together with the precontrast and postcontrast vessel wall images were used for contrast enhancement assessment [12]. In addition to contrast enhancement, specific lesion characteristics (configuration and thickening pattern) were also assessed [see Electronic supplementary material (ESM)].

\section{Statistical analysis}

Differences between image quality ratings at $3 \mathrm{~T}$ and $7 \mathrm{~T}$ were calculated using a non-parametric Wilcoxon signed-rank test. 
Inter-rater agreement of the number and location of vessel wall lesions was evaluated using the Dice's similarity coefficient (DSC) [23] for the 3-T and 7-T MR images separately. For inter-rater agreement of contrast enhancement assessment Cohen's kappa was calculated. Statistical analyses were performed using IBM SPSS Statistics (version 21; IBM Corporation, Armonk, NY, USA). A $p$ value of $<0.05$ was considered to be statistically significant. Bonferroni correction for multiple comparisons was applied when appropriate.

\section{Results}

\section{Study population}

Twenty-one healthy volunteers (12 men; age $66 \pm 5$ years) were included. Baseline characteristics are shown in ESM Table I.

\section{Image quality}

Since image quality ratings of both raters were comparable, the mean of both raters was used. Seven-tesla images showed significantly more artefacts compared with $3 \mathrm{~T}$, both precontrast and postcontrast (Table 2). For analysis of vessel wall visibility, subjects scored with artefacts hampering assessment on either 3-T or 7-T images where the majority of the vessel walls were affected (i.e. due to severe motion artefacts) were excluded ( $n=6$; motion artefacts at $3 \mathrm{~T}(n=2)$ or 7 $\mathrm{T}(n=4)$; precontrast $n=2$ and postcontrast $n=4)$. Overall vessel wall visibility was scored significantly better for the 7-T images compared with $3 \mathrm{~T}$, both precontrast and postcontrast (Table 2). On arterial segment level (Table 3), the ICA, ACA and proximal MCA (M1 segment) vessel walls were significantly better visible at $7 \mathrm{~T}$; this was also the case for the P2 segment.

\section{Vessel-wall lesions}

For analysis of vessel-wall lesions, subjects with poor overall vessel wall visibility scored by both raters on either 3-T or 7-T precontrast images were excluded $[n=5$; poor visibility at $3 \mathrm{~T}$ only $(n=1), 7 \mathrm{~T}$ only $(n=2)$, or both at $3 \mathrm{~T}$ and $7 \mathrm{~T}(n=2)]$, resulting in 16 subjects for analysis. For analysis of lesion enhancement, an additional three subjects were excluded based on poor overall visibility of the postcontrast scan [poor visibility at 3 T only $(n=2)$ or 7 T only $(n=1)]$.

Pre-consensus inter-rater agreement for number and location of the identified vessel-wall lesions was good at both $3 \mathrm{~T}$ (DSC, 0.68) and $7 \mathrm{~T}$ (DSC, 0.67). Also, a good to excellent
Table 2 Qualitative scoring of artefacts and overall visibility of the arterial vessel wall on 3$\mathrm{T}$ and 7-T MRI (precontrast and postcontrast)

\begin{tabular}{|c|c|c|c|c|c|c|}
\hline & \multicolumn{3}{|l|}{ Precontrast } & \multicolumn{3}{|c|}{ Postcontrast } \\
\hline & $3 \mathrm{~T}$ & $7 \mathrm{~T}$ & $p$-value ${ }^{\mathrm{c}}$ & $3 \mathrm{~T}$ & $7 \mathrm{~T}$ & $p$-value ${ }^{c}$ \\
\hline \multicolumn{7}{|l|}{ Artefacts $^{\mathrm{a}}$} \\
\hline 0 & $2(5 \%)$ & $14(33 \%)$ & & $6(14 \%)$ & $16(38 \%)$ & \\
\hline 1 & $29(69 \%)$ & $28(67 \%)$ & & $32(76 \%)$ & $26(62 \%)$ & \\
\hline 2 & $11(26 \%)$ & $0(0 \%)$ & & $4(10 \%)$ & $0(0 \%)$ & \\
\hline Proportion of overall agreement (\%) & 76 & 62 & & 81 & 71 & \\
\hline Mean rater 1 (range) & $1.24(0-2)$ & $0.57(0-1)$ & & $0.95(0-2)$ & $0.57(0-1)$ & \\
\hline Mean rater 2 (range) & $1.19(0-2)$ & $0.76(0-1)$ & & $0.95(0-2)$ & $0.67(0-1)$ & \\
\hline Mean both raters & 1.21 & 0.67 & $<0.001$ & 0.95 & 0.62 & 0.024 \\
\hline \multicolumn{7}{|l|}{ Overall visibility vessel wall $^{\mathrm{b}}$} \\
\hline 0 & $5(17 \%)$ & $3(10 \%)$ & & $5(17 \%)$ & $3(10 \%)$ & \\
\hline 1 & $22(73 \%)$ & $11(37 \%)$ & & $23(77 \%)$ & $14(47 \%)$ & \\
\hline 2 & $3(10 \%)$ & $16(53 \%)$ & & $2(7 \%)$ & $13(43 \%)$ & \\
\hline Proportion of overall agreement (\%) & 60 & 80 & & 80 & 87 & \\
\hline Mean rater 1 (range) & $0.87(0-2)$ & $1.40(0-2)$ & & $0.93(0-2)$ & $1.33(0-2)$ & \\
\hline Mean rater 2 (range) & $1.00(0-2)$ & $1.47(0-2)$ & & $0.87(0-2)$ & $1.33(0-2)$ & \\
\hline Mean both raters & 0.93 & 1.43 & 0.009 & 0.90 & 1.33 & 0.019 \\
\hline
\end{tabular}

Grading scale overall artefacts: $0=$ artefacts hampering assessment; $1=$ moderate artefacts, but images assessable; $2=$ no artefacts

Grading scale overall visibility of the arterial vessel wall: $0=$ poor; $1=$ moderate; $2=\operatorname{good}$

${ }^{a}$ Based on 21 subjects

${ }^{\mathrm{b}}$ Based on 15 subjects

${ }^{\mathrm{c}}$ Bonferroni corrected significance level $p<0.025$ (corrected for two comparisons of rating scales) 
Table 3 Qualitative visibility scoring of all separate arterial vessel wall segments of the circle of Willis and its primary branches on 3-T and 7-T MRI (precontrast) ${ }^{\mathrm{a}}$

\begin{tabular}{|c|c|c|c|}
\hline Location & $3 \mathrm{~T}$ & $7 \mathrm{~T}$ & $p$-value ${ }^{\mathrm{b}}$ \\
\hline \multicolumn{4}{|l|}{ Anterior cerebral artery } \\
\hline A1 segment & $2.21(1-3)$ & $2.79(2-4)$ & $0.001 *$ \\
\hline A2 segment & $1.63(1-3)$ & $2.24(1-4)$ & $<0.001 *$ \\
\hline Anterior communicating artery & $1.16(1-2)$ & $1.32(1-3)$ & 0.398 \\
\hline \multicolumn{4}{|l|}{ Middle cerebral artery } \\
\hline M1 segment & $2.11(1-3)$ & $2.76(2-4)$ & $<0.001 *$ \\
\hline M2 segment & $2.16(1-3)$ & $2.24(1-3)$ & 0.802 \\
\hline M3 segment & $1.34(0-3)$ & $1.05(0-2)$ & $0.052^{\mathrm{c}}$ \\
\hline \multicolumn{4}{|l|}{ Internal carotid artery } \\
\hline Distal intracranial segment & $2.82(2-3)$ & $3.37(2-4)$ & $0.002 *$ \\
\hline Intracranial bifurcation & $2.61(2-4)$ & $3.16(2-4)$ & $<0.001 *$ \\
\hline Posterior communicating artery & $1.90(1-3)$ & $2.50(1-4)$ & 0.083 \\
\hline \multicolumn{4}{|l|}{ Posterior cerebral artery } \\
\hline P1 segment & $2.55(1-4)$ & $3.13(2-4)$ & 0.003 \\
\hline Bifurcation & $2.26(1-4)$ & $3.08(2-4)$ & 0.004 \\
\hline P2 segment & $1.79(1-3)$ & $2.63(2-3)$ & $<0.001 *$ \\
\hline \multicolumn{4}{|l|}{ Basilar artery } \\
\hline Bifurcation & $2.74(1-4)$ & $2.90(1-4)$ & 0.413 \\
\hline Distal half & $2.97(2-4)$ & $2.90(1-4)$ & 0.499 \\
\hline Proximal half & $3.11(1-4)$ & $2.97(1-4)$ & 0.545 \\
\hline \multicolumn{4}{|l|}{ Vertebral artery } \\
\hline Distal half & $3.26(2-4)$ & $2.84(1-4)$ & 0.168 \\
\hline Proximal half & $3.32(2-4)$ & $2.97(1-4)$ & 0.214 \\
\hline
\end{tabular}

*statistically significant (after Bonferroni correction)

Scores are given as mean (range) of both raters

Grading scale: $0=$ outside FOV; $1=$ not visible; $2=$ poor; $3=$ moderate; $4=$ good

${ }^{\text {a }}$ Based on 19 subjects per location

${ }^{\mathrm{b}}$ Bonferroni corrected significance level $p<0.003$ (corrected for 17 comparisons of arterial segments)

${ }^{c}$ Rating " 0 " was excluded from statistical analysis ( $n=3$ subjects)

inter-rater agreement was found for lesion contrast enhancement (kappa 0.85 for $3 \mathrm{~T}$, and 0.67 for $7 \mathrm{~T}$ ). On the precontrast scans, 45 (at $3 \mathrm{~T}$ ) and 67 (at $7 \mathrm{~T}$ ) vessel-wall lesions were scored after consensus; an additional 3 (at $3 \mathrm{~T}$ ) and 12 (at 7 T) lesions were identified because of vessel-wall enhancement. In total, 48 vessel-wall lesions were identified at $3 \mathrm{~T}$ (mean, 3 per subject; range, 1-7), of which 7 (15\%) showed enhancement (Table 4). At 7 T, 79 lesions were identified (mean, 5 per subject; range, 1-10), of which 29 (37\%) showed enhancement (Table 4).

\section{Side-by-side comparison}

Twenty-four vessel wall lesions were identified at both $3 \mathrm{~T}$ and 7 T (Figs. 1 and 2). Almost one-third (7/24; $29 \%$ ) of lesions visible at both $3 \mathrm{~T}$ and $7 \mathrm{~T}$ showed enhancement on both field strengths; an additional one-third $(9 / 24 ; 38 \%)$ showed enhancement at $7 \mathrm{~T}$ only. Most corresponding lesions were present in the vertebral arteries, basilar artery and internal carotid arteries.

Side-by-side comparison of the non-corresponding vesselwall lesions showed that in retrospect, 10 lesions $(21 \%)$ scored only at $3 \mathrm{~T}$ and 23 lesions (29\%) scored only at $7 \mathrm{~T}$ were either visible retrospectively but missed at the other field strength by both raters, or identified but omitted during the consensus reading (Fig. 3). Fourteen (29\%) vessel-wall lesions that were identified at $3 \mathrm{~T}$ could not be retrospectively identified at 7 T. At 7 T, 32 (41\%) vessel wall lesions could not be retrospectively identified at $3 \mathrm{~T}$ (Fig. 4). The noncorresponding lesions were mainly located in areas where the vessel wall was less well visible; also, several of these lesions appeared smaller compared with the corresponding lesions. In total, 34 (71 \%) of all lesions identified at $3 \mathrm{~T}(24$ after consensus +10 in retrospect) and $47(59 \%)$ at $7 \mathrm{~T}(24$ after consensus +23 in retrospect) were also seen on the other field strength.

\section{Discussion}

In the current study, vessel wall visibility was equal or significantly better at $7 \mathrm{~T}$ compared with $3 \mathrm{~T}$ for the studied arterial segments, even though there were more artefacts hampering assessment at $7 \mathrm{~T}$. Furthermore, more vessel wall lesions were scored on 7-T images. However, surprisingly, only $71 \%$ of all 3-T lesions were seen at $7 \mathrm{~T}$.

Better visualisation of the vessel wall at $7 \mathrm{~T}$ was most prominent in the proximal anterior cerebral circulation and the P2 segment of the PCA; the basilar artery and vertebral arteries were visualised equally good at both field strengths. The latter could be explained by better CSF suppression in this region compared to other brain regions at $3 \mathrm{~T}$, probably because of higher local CSF flow pulsation. Artefacts at $7 \mathrm{~T}$ were mainly caused by motion artefacts or signal loss and $\mathrm{B}_{1}{ }^{+}$inhomogeneities in the areas of the temporal lobes and cerebellum. Although motion artefacts were also present at $3 \mathrm{~T}$, they occurred more frequently at $7 \mathrm{~T}$. This might be related to the longer acquisition time of the 7-T sequence compared with 3 $\mathrm{T}$, increasing the possibility of subject motion during scanning [24]. $\mathrm{B}_{1}{ }^{+}$inhomogeneities at $7 \mathrm{~T}$ result in a spatially varying SNR and contrast that is most pronounced at the temporal lobes and cerebellum $[13,16]$. In this study, dielectric bags were placed in the upper neck region of the subjects to improve imaging of the cerebellar region, which improved part of the signal loss, but did not reduce signal loss in all subjects.

Most vessel wall lesions were found using $7 \mathrm{~T}$, which was expected since CSF suppression is better at $7 \mathrm{~T}$ than at $3 \mathrm{~T}$ in most brain regions. At $3 \mathrm{~T}$, no explicit CSF suppression can be 
Table 4 Overview of number, location and enhancement of identified vessel wall lesions on 3-T and 7-T vessel wall images, as well as lesions that corresponded between $3 \mathrm{~T}$ and $7 \mathrm{~T}$

\begin{tabular}{|c|c|c|c|c|c|c|c|}
\hline \multirow[b]{3}{*}{ Location } & \multicolumn{2}{|l|}{$3 \mathrm{~T}$} & \multicolumn{2}{|l|}{$7 \mathrm{~T}$} & \multicolumn{3}{|c|}{ Corresponding $3 \mathrm{~T}-7 \mathrm{~T}$} \\
\hline & \multirow[t]{2}{*}{ Lesions } & \multirow[t]{2}{*}{ Enhancement $^{\mathrm{a}}$} & \multirow[t]{2}{*}{ Lesions } & \multirow[t]{2}{*}{ Enhancement $^{\mathrm{b}}$} & \multirow[t]{2}{*}{ Lesions } & \multicolumn{2}{|c|}{ Enhancement $^{\mathrm{c}}$} \\
\hline & & & & & & $3 \mathrm{~T}$ & $7 \mathrm{~T}$ \\
\hline Total anterior circulation & $17(35.4)$ & 1 & $32(40.5)$ & 8 & 7 & 1 & 4 \\
\hline Anterior cerebral artery & $3(6.3)$ & 0 & $3(3.8)$ & 0 & 0 & 0 & 0 \\
\hline A1 segment & $2(4.2)$ & 0 & $1(1.3)$ & 0 & 0 & 0 & 0 \\
\hline A2 segment & $1(2.1)$ & 0 & $2(2.5)$ & 0 & 0 & 0 & 0 \\
\hline Anterior communicating artery & $0(0.0)$ & 0 & $0(0.0)$ & 0 & 0 & 0 & 0 \\
\hline Middle cerebral artery & $6(12.5)$ & 1 & $13(16.5)$ & 2 & 3 & 1 & 2 \\
\hline M1 segment & $5(10.4)$ & 1 & $9(11.4)$ & 2 & 3 & 1 & 2 \\
\hline M2 segment & $1(2.1)$ & 0 & $4(5.1)$ & 0 & 0 & 0 & 0 \\
\hline M3 segment & $0(0.0)$ & 0 & $0(0.0)$ & 0 & 0 & 0 & 0 \\
\hline Internal carotid artery & $8(16.7)$ & 0 & $16(20.3)$ & 6 & 4 & 0 & 2 \\
\hline Distal intracranial segment & $3(6.3)$ & 0 & $6(7.6)$ & 3 & 1 & 0 & 1 \\
\hline Intracranial bifurcation & $5(10.4)$ & 0 & $10(12.7)$ & 3 & 3 & 0 & 1 \\
\hline Total posterior circulation & $31(64.6)$ & 6 & $47(59.5)$ & 21 & 17 & 6 & 12 \\
\hline Posterior communicating artery & $2(4.2)$ & 0 & $2(2.5)$ & 0 & 1 & 0 & 0 \\
\hline Posterior cerebral artery & $4(8.3)$ & 0 & $11(13.9)$ & 1 & 1 & 0 & 0 \\
\hline P1 segment & $2(4.2)$ & 0 & $0(0.0)$ & 0 & 0 & 0 & 0 \\
\hline Bifurcation & $0(0.0)$ & 0 & $4(5.1)$ & 0 & 0 & 0 & 0 \\
\hline P2 segment & $2(4.2)$ & 0 & $7(8.9)$ & 1 & 1 & 0 & 0 \\
\hline Basilar artery & $8(16.7)$ & 0 & $10(12.7)$ & 3 & 4 & 0 & 2 \\
\hline Bifurcation & $5(10.4)$ & 0 & $5(6.3)$ & 1 & 2 & 0 & 1 \\
\hline Distal half & $3(6.3)$ & 0 & $2(2.5)$ & 1 & 2 & 0 & 1 \\
\hline Proximal half & $0(0.0)$ & 0 & $3(3.8)$ & 1 & 0 & 0 & 0 \\
\hline Vertebral artery & $17(35.4)$ & 6 & $24(30.4)$ & 17 & 11 & 6 & 10 \\
\hline Distal half & $3(6.3)$ & 0 & $6(7.6)$ & 3 & 1 & 0 & 1 \\
\hline Proximal half & $14(29.2)$ & 6 & $18(22.8)$ & 14 & 10 & 6 & 9 \\
\hline Total & 48 & 7 & 79 & 29 & 24 & 7 & 16 \\
\hline
\end{tabular}

Number of lesions per location ( $\%$ from total)

${ }^{a} n=7$ lesions not assessable on postcontrast scans

${ }^{\mathrm{b}} n=13$ lesions not assessable on postcontrast scans

${ }^{\mathrm{c}} n=3$ lesions not assessable on postcontrast scans

performed; therefore, suppression depends on the amount of CSF flow during the spin echo train which is spatially dependent, resulting in varying quality of CSF suppression in different brain regions. The 7-T sequence contains a nonselective adiabatic inversion pulse for global CSF suppression. Most corresponding lesions between $3 \mathrm{~T}$ and $7 \mathrm{~T}$ were present in the vertebral arteries, basilar artery, and internal carotid arteries: all larger arterial segments of the circle of Willis around which considerable CSF flow pulsation is present. These segments scored relatively good for vessel wall visibility at both $3 \mathrm{~T}$ and $7 \mathrm{~T}$.

Close to one-half of 7-T lesions ( $41 \%$ ) could not be identified at 3-T MRI. On the other hand, a striking one-third
(29\%) of all lesions identified at $3 \mathrm{~T}$ were not visible at $7 \mathrm{~T}$. The latter might be explained by the higher acquired in-plane spatial resolution of the 3 -T sequence $\left(0.6 \times 0.6 \mathrm{~mm}^{2}\right)$ compared with the 7-T sequence $\left(0.8 \times 0.8 \mathrm{~mm}^{2}\right)$, so that very small lesions seen at $3 \mathrm{~T}$ might have been missed at $7 \mathrm{~T}$. The first could be explained by the increased SNR and CNR as well as better CSF suppression at $7 \mathrm{~T}$. Another possible explanation, applicable to both findings, could be that for smaller lesions we are currently at the edge of what can be visualised with the used vessel wall imaging sequences. For these small lesions, scan parameter differences between both field strengths (e.g. slice thickness, voxel sizes) as well as field inhomogeneities may become more important [25]. 
Fig. 1 Corresponding vesselwall lesions at $3 \mathrm{~T}$ and $7 \mathrm{~T}$ (arrows) on the precontrast images: located at the left distal intracranial segment of the ICA

(a), right $\mathrm{P} 2$ segment of the PCA (b) and left M1 segment of the MCA (c). ICA intracranial internal carotid artery, $M C A$ middle cerebral artery, $P C A$ posterior cerebral artery

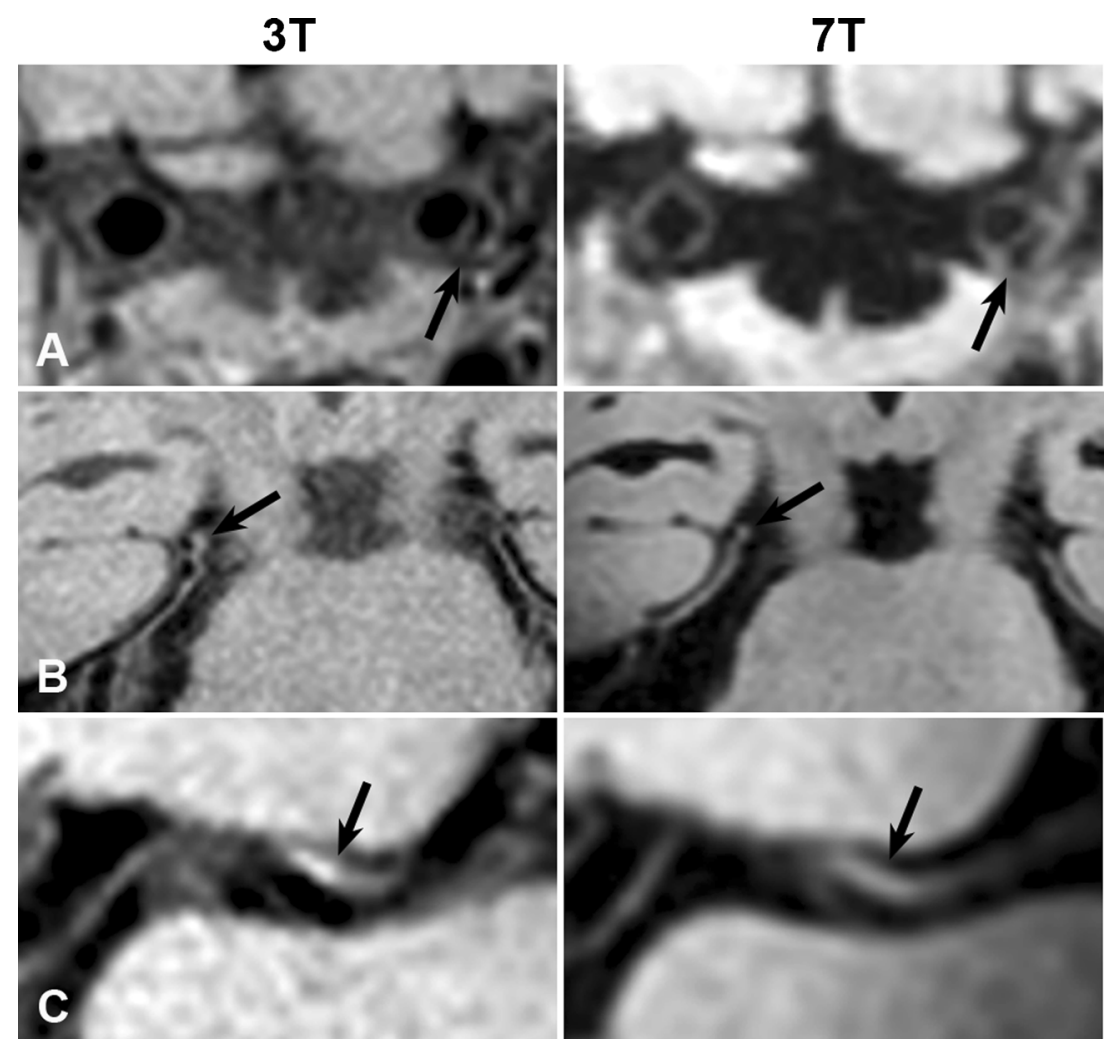

Consequently, both SNR and CNR may become too low to reliably differentiate lesions from noise, resulting in more subjective assessment. Also, there might be a higher risk of misinterpreting vessel wall irregularities caused by other phenomena like slow flow directly alongside the artery mimicking a lesion, or incomplete CSF suppression [11, 26, 27]. In

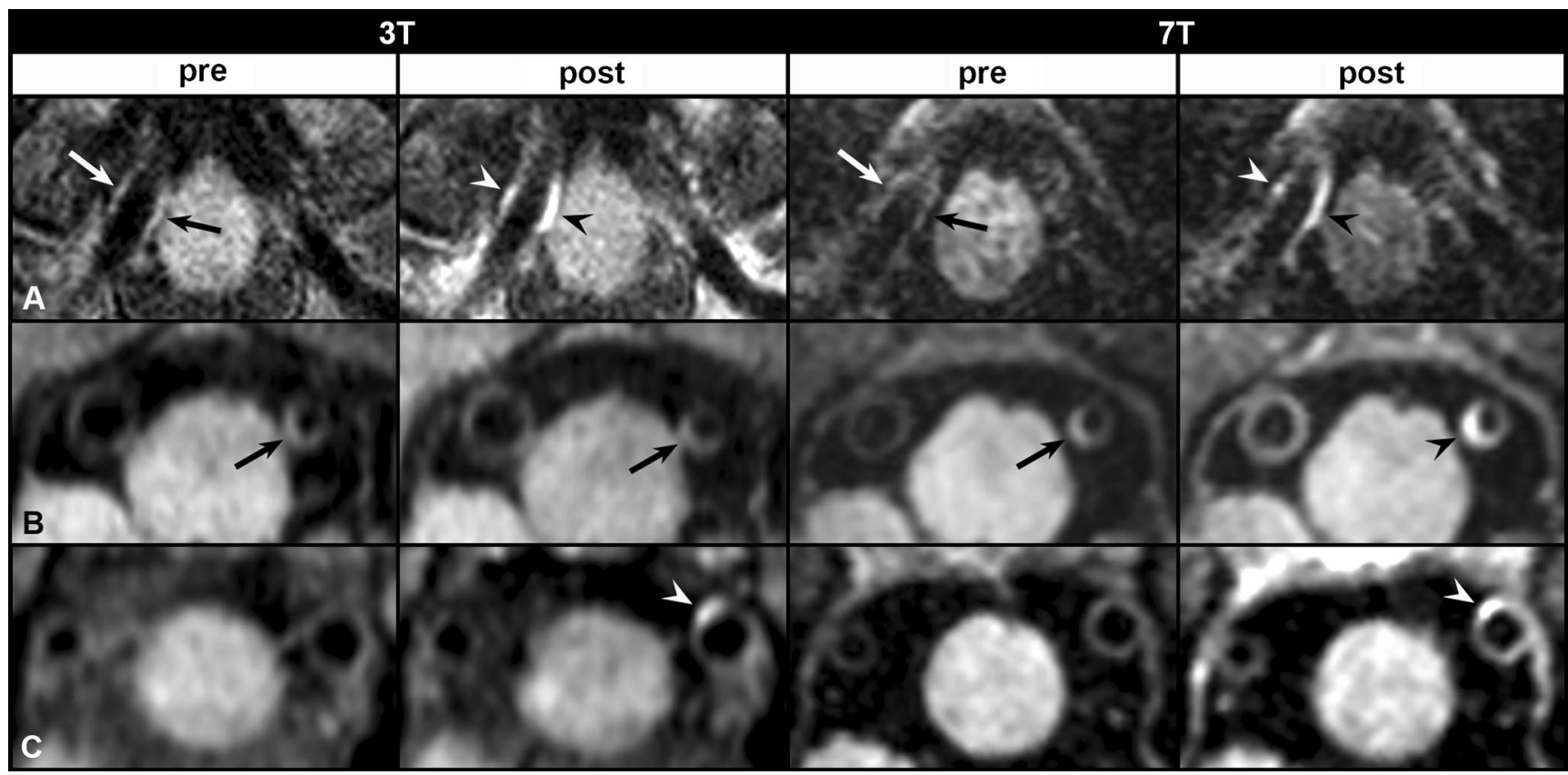

Fig. 2 Vessel-wall enhancement after contrast administration. a, b Vessel-wall lesions identified on the precontrast $3 \mathrm{~T}$ and $7 \mathrm{~T}$ vessel wall images (arrows), with enhancement (arrowheads) on the postcontrast images at $3 \mathrm{~T}$ and $7 \mathrm{~T}$ (a right proximal VA), and $7 \mathrm{~T}$ only (b left proximal VA). c Vessel wall lesion identified based on enhancement of the vessel wall on the postcontrast $3 \mathrm{~T}$ and $7 \mathrm{~T}$ vessel wall images (c left proximal VA). VA vertebral artery 
Fig. 3 Non-corresponding intracranial vessel wall lesions (arrows or arrowheads) identified on either the 3-T $(\mathbf{a}, \mathbf{b})$ or 7-T (c, d) vessel wall images that were identified retrospectively on the other scan: located at the right $\mathrm{P} 1$ segment of the PCA (a), bifurcation BA-P1 (b), right bifurcation P1-P2 segment of the PCA (c), and right M1 segment of the MCA (d). $B A$ basilar artery, $M C A$ middle cerebral artery, $P C A$ posterior cerebral artery
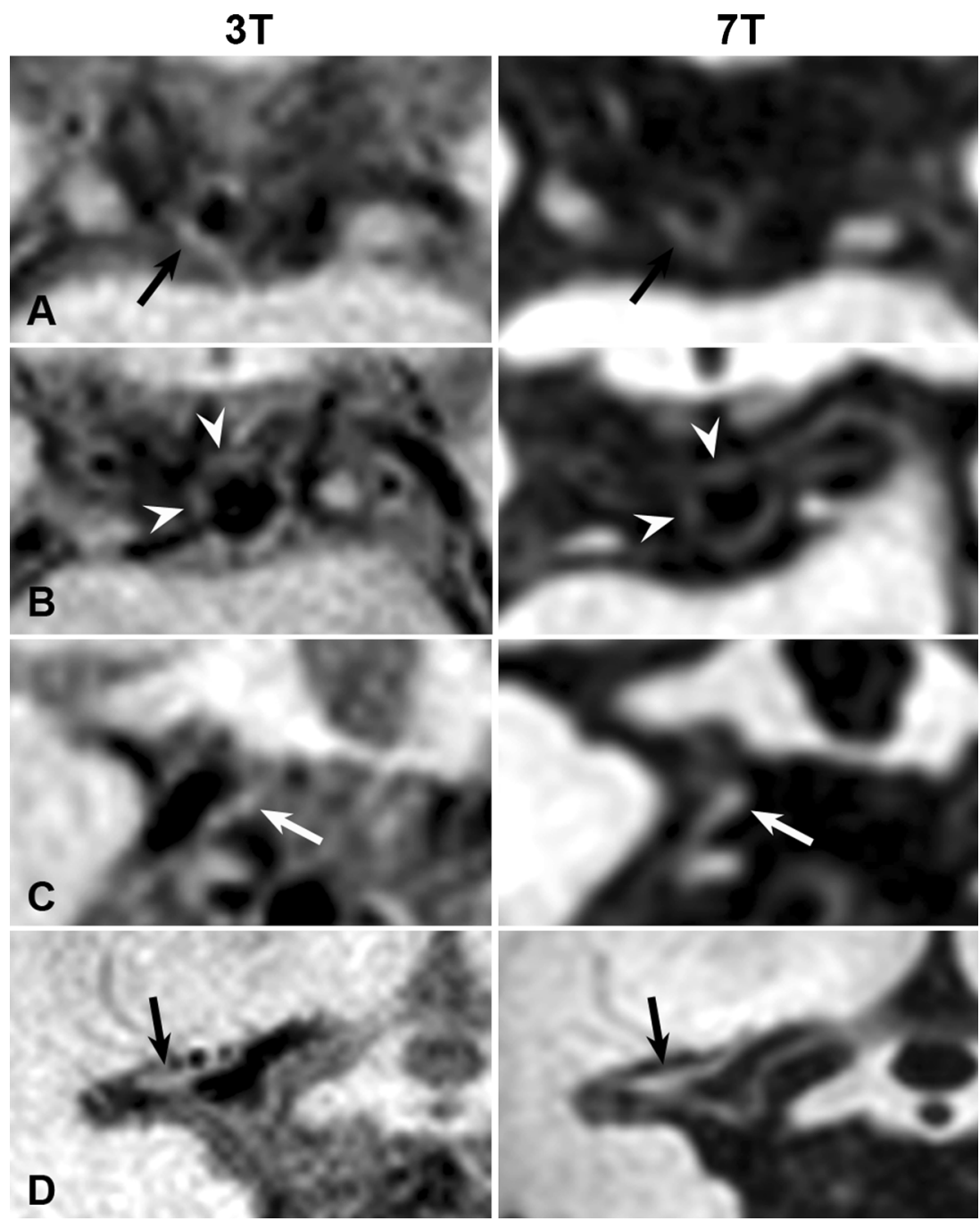

addition, we can contemplate whether these wall thickenings might actually reflect normal thickness variation throughout the intracranial arterial vasculature. Although two recent postmortem studies have shown that ultrahigh-resolution 7-T MRI can identify atherosclerotic plaques in intracranial arteries $[14$, 28], validation of in vivo MRI results with histology has not yet been performed. Therefore, we do not really know to what extent all identified vessel wall lesions in this study are true atherosclerotic lesions, especially when these lesions are seen on one field strength only. This makes it difficult to determine which field strength shows vessel wall lesions best.

One striking secondary result is that in the elderly asymptomatic population included in this study, a substantial amount of intracranial vessel-wall lesions have been found. Most studies have attempted to target symptomatic intracranial atherosclerosis; until now, only transcranial Doppler has been used to assess the presence of intracranial atherosclerosis in an asymptomatic population [29], but this technique solely provides information about the presence of stenotic lesions, i.e. advanced atherosclerotic lesions. Previous studies in a symptomatic patient population with ischaemic stroke/TIA imaged with the same 7-T MRI sequence found $84 \%$ of patients had on average three vessel-wall lesions in the circle of Willis arteries, and $100 \%$ of patients had on average four lesions if the vertebral arteries were also (mostly) taken into account $[19,21]$. These results show a striking similarity with our current results of $100 \%$ of subjects with on average five vessel-wall lesions at $7 \mathrm{~T}$; however, our study population consists of asymptomatic healthy elderly volunteers instead of patients with symptomatic cerebrovascular disease.

Another striking result is that in our elderly asymptomatic population, many of the intracranial vessel-wall lesions showed enhancement after contrast administration, predominantly at $7 \mathrm{~T}$. Shortening of the $\mathrm{T}_{1}$ relaxation time caused by the injected contrast agent is suggested to be reduced at higher field strengths [30]. However, the increased SNR at $7 \mathrm{~T}$ may theoretically make the sequence more sensitive for smaller amounts of contrast agent, e.g. when present in a vessel wall lesion. Also, the 7-T sequence used in this study might be stronger $\mathrm{T}_{1}$-weighted than the 3-T sequence, making the 7-T 
Fig. 4 Non-corresponding intracranial vessel-wall lesions (arrows) identified on either the 3-T $(\mathbf{a}, \mathbf{b})$ or 7-T (c, d) vessel-wall images that could also not be identified on the other field strength retrospectively: located at the bifurcation BA-P1 (a visible at $3 \mathrm{~T}$ ), left $\mathrm{M} 2$ segment of the MCA (b visible at $3 \mathrm{~T}$ ), left M1 segment of the MCA (c visible at $7 \mathrm{~T}$ ) and left distal intracranial segment of the ICA (d visible at $7 \mathrm{~T}) . B A$ basilar artery, $I C A$ internal carotid artery, $M C A$ middle cerebral artery
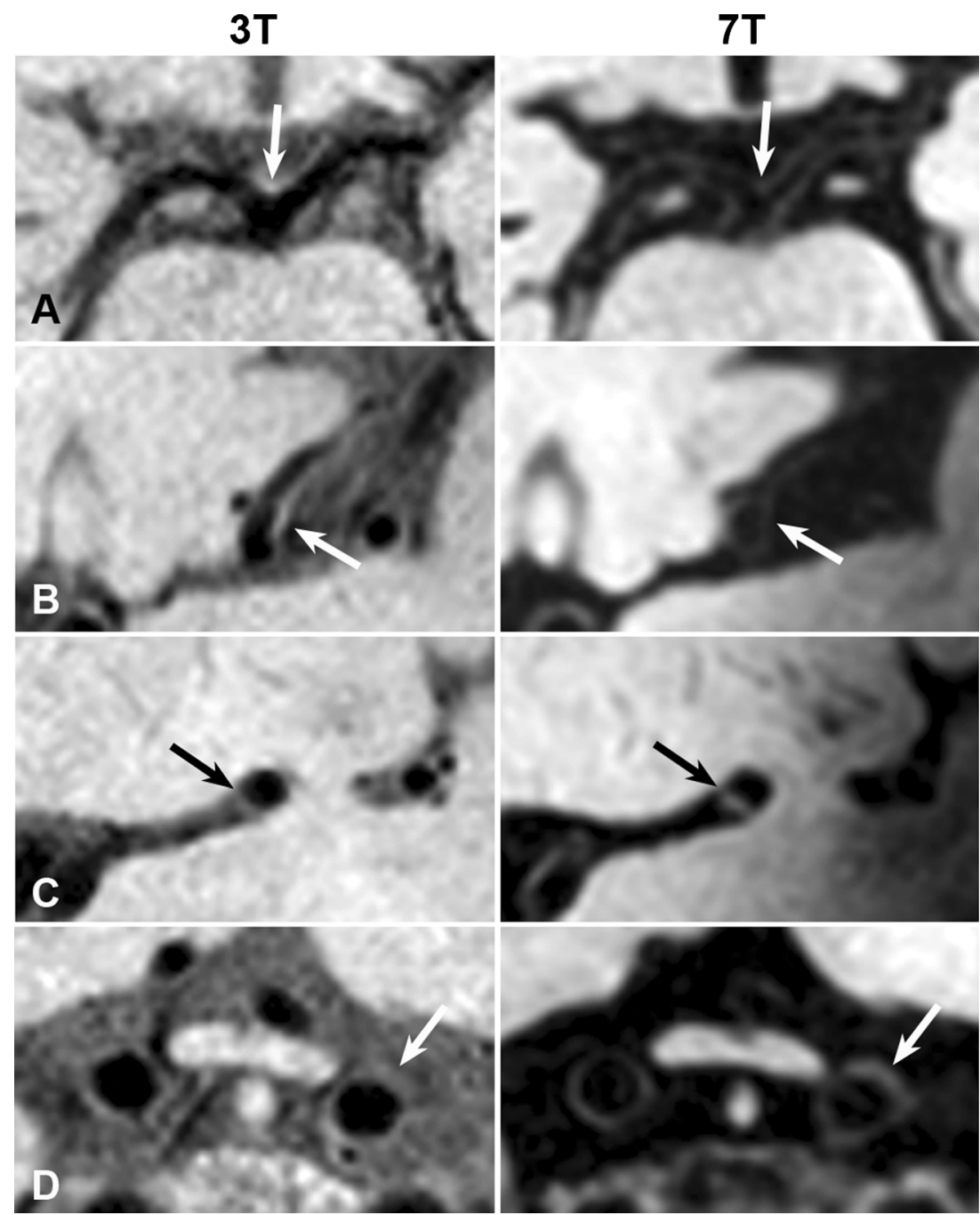

sequence more sensitive for visualising enhancement. Another possible explanation might be that the time between contrast administration and acquisition of the postcontrast vessel-wall scan was not exactly identical during both MRI examinations [8] (approximately $4 \mathrm{~min}$ shorter at $3 \mathrm{~T}$ ). Contrast enhancement of intracranial atherosclerotic plaques has been associated with acute ischaemic stroke [31-33] and could be a marker for plaque inflammation or neovascularisation [34], and even potentially of intracranial plaque instability and stroke risk [33]. Our results, however, show that enhancing lesions are not always associated with (acute) ischaemic stroke or plaque inflammation.

The results from this study shed new light on the clinical application of intracranial vessel wall imaging, and raise important issues that need to be considered. Currently, differentiation between atherosclerotic lesions and normal vessel-wall thickness variations resp. (CSF) flow artefacts alongside the artery walls is difficult. Although much is known already about in vivo vessel-wall (intima-media) thickness of extracranial arteries [35], limited information is available on (normal) vessel-wall thickness variations of the arteries of the circle of Willis. The current study shows vessel-wall lesion burden of the asymptomatic population was comparable to that of symptomatic patients in previous studies. Apart from being vessel-wall lesions, these wall thickenings could also be a reflection of 'normal' variation in vessel wall thickening throughout the intracranial arterial vasculature. Therefore, the clinical relevance of these vessel-wall lesions might be limited. This may be especially true for enhancing lesions that, based on our current study, are apparently not always associated with ischaemic stroke or plaque inflammation, as has thus far been assumed. Therefore, future studies comparing both symptomatic and asymptomatic patients should be performed, to gain more insight into differences of vessel-wall lesion burden/thickness variations, as well as contrast enhancement of the vessel wall.

The current study uses a 7-T vessel wall sequence with a relatively low spatial resolution compared with most published (and our own) 3-T sequence(s). A disadvantage of using an inversion recovery pulse (for optimal CSF suppression) is 
that it increases acquisition time. Therefore, the acquired spatial resolution of the 7-T sequence used in our study is currently restricted to $0.8 \times 0.8 \times 0.8 \mathrm{~mm}^{3}$. As a consequence, some of the scored vessel wall lesions will be smaller than the true voxel size of this 7-T sequence (although this is also true for most used 3-T sequences), since the intracranial vessel wall is typically very thin. However, a previous study by Kleinloog et al. [36], in which the same 7-T vessel-wall sequence was used as in the current study, showed that signal intensity variation of the vessel wall reflects thickness variation. Therefore, it is possible to infer thickness variations from the 7-T vessel-wall images even for vessel walls that are thinner than the acquired voxel size. Also, the advantage of high image contrast between vessel wall (lesions) and surrounding tissue at $7 \mathrm{~T}$, due to the applied inversion recovery pulse, could compensate for, or be even more important than, spatial resolution for visualising vessel wall lesions. Although other research groups have reported vessel wall sequences with higher resolutions, they achieved less optimal CSF suppression [10, 11, 37, 38]. Future work should be done to evaluate the relative importance of resolution versus contrast and CSF suppression.

This study has several limitations. First, the MRI sequences used at both field strengths were not exactly the same: the main differences were the higher in-plane spatial resolution of the 3-T sequence and higher through-plane (slice thickness) spatial resolution of the 7-T sequence, and the lack of an inversion pulse for CSF suppression at $3 \mathrm{~T}$. However, to achieve a fair comparison between both field strengths, we found using optimised imaging sequences for each field strength the best option. Second, the receive coils used in this study were different (eight-channel head coil at $3 \mathrm{~T}$ versus 32channel head coil at $7 \mathrm{~T}$ ). Third, in this study all minor vessel wall thickness variations were scored as vessel wall lesions. As discussed earlier, especially for small vessel wall lesions, we cannot be certain that these are all actual vessel wall lesions; for translation of the vessel wall imaging findings to clinical practice, as well as usability in future studies, validation with a "gold standard" (histology) is a prerequisite. Fourth, blinding the raters for field strength was not possible due to the inherent slightly different image contrast between 3 $\mathrm{T}$ and $7 \mathrm{~T}$; this might have induced a bias in image rating. Fifth, the sample size of this study was relatively small. However, this is the first study to provide a comparison of 3$\mathrm{T}$ and 7-T intracranial vessel-wall imaging, as well as vesselwall imaging in an asymptomatic elderly population with the administration of a contrast agent to assess enhancement of the vessel wall. Lastly, although 7-T MRI is still less available compared with the lower field strength MR systems, the number of installed 7-T MR systems has increased in recent years [39]. Together with the tremendous growth of technical developments at 7-T MRI over the last decade to overcome difficulties of imaging at higher field strength, its use may become more broadly available in the near future.
In summary, in this comparative study of a dedicated 3-T VIRTA and 7-T MPIR-TSE intracranial vessel-wall sequence in asymptomatic individuals, 7-T vessel-wall imaging appears to have the highest potential to identify the total burden of vessel wall lesions in the arteries of the circle of Willis and its branches. However, a direct comparison between symptomatic and asymptomatic patients is warranted to elucidate the potential for clinical application of intracranial vessel-wall imaging.

Acknowledgments The scientific guarantor of this publication is Prof. J. Hendrikse. The authors of this manuscript declare no relationships with any companies, whose products or services may be related to the subject matter of the article. This study has received funding from the Netherlands Organisation for Scientific Research (NWO) under grant no. 91712322 (J.H.), the European Research Council under grant no. 637024 (J.H.) and no. 337333 (J.J.M.Z.) and the Dutch Heart Foundation under grant no. 2010T075 (H.B.v.d.W.). No complex statistical methods were necessary for this paper. Institutional Review Board approval was obtained. Written informed consent was obtained from all subjects in this study. Methodology: retrospective, observational, performed at one institution.

Open Access This article is distributed under the terms of the Creative Commons Attribution 4.0 International License (http:// creativecommons.org/licenses/by/4.0/), which permits unrestricted use, distribution, and reproduction in any medium, provided you give appropriate credit to the original author(s) and the source, provide a link to the Creative Commons license, and indicate if changes were made.

\section{References}

1. Arenillas JF (2011) Intracranial atherosclerosis: current concepts. Stroke 42:S20-S23

2. Qureshi AI, Caplan LR (2014) Intracranial atherosclerosis. Lancet 383:984-998

3. Mazighi M, Labreuche J, Gongora-Rivera F, Duyckaerts C, Hauw JJ, Amarenco P (2008) Autopsy prevalence of intracranial atherosclerosis in patients with fatal stroke. Stroke 39:1142-1147

4. Glagov S, Weisenberg E, Zarins CK, Stankunavicius R, Kolettis GJ (1987) Compensatory enlargement of human atherosclerotic coronary arteries. N Engl J Med 316:1371-1375

5. Ward MR, Pasterkamp G, Yeung AC, Borst C (2000) Arterial remodeling. Mechanisms and clinical implications. Circulation 102: 1186-1191

6. Leng X, Wong KS, Liebeskind DS (2014) Evaluating intracranial atherosclerosis rather than intracranial stenosis. Stroke 45:645-651

7. Bodle JD, Feldmann E, Swartz RH, Rumboldt Z, Brown T, Turan TN (2013) High-resolution magnetic resonance imaging: an emerging tool for evaluating intracranial arterial disease. Stroke 44:287292

8. Dieleman N, van der Kolk AG, Zwanenburg JJ et al (2014) Imaging intracranial vessel wall pathology with magnetic resonance imaging: current prospects and future directions. Circulation 130:192201

9. Koops A, Ittrich H, Petri S et al (2007) Multicontrast-weighted magnetic resonance imaging of atherosclerotic plaques at 3.0 and 1.5 Tesla: ex-vivo comparison with histopathologic correlation. Eur Radiol 17:279-286 
10. Qiao Y, Steinman DA, Qin Q et al (2011) Intracranial arterial wall imaging using three-dimensional high isotropic resolution black blood MRI at 3.0 Tesla. J Magn Reson Imaging 34:22-30

11. Wang J, Helle M, Zhou Z, Bornert P, Hatsukami TS, Yuan C (2016) Joint blood and cerebrospinal fluid suppression for intracranial vessel wall MRI. Magn Reson Med 75:831-838

12. van der Kolk AG, Zwanenburg JJ, Brundel M et al (2011) Intracranial vessel wall imaging at 7.0-T MRI. Stroke 42:24782484

13. van der Kolk AG, Hendrikse J, Zwanenburg JJ, Visser F, Luijten PR (2013) Clinical applications of 7 T MRI in the brain. Eur J Radiol 82:708-718

14. Harteveld AA, Denswil NP, Siero JC et al (2015) Quantitative intracranial atherosclerotic plaque characterization at 7T MRI: an ex vivo study with histologic validation. AJNR Am J Neuroradiol $37: 802-810$

15. van der Kolk AG, Hendrikse J, Brundel M et al (2013) Multisequence whole-brain intracranial vessel wall imaging at 7.0 tesla. Eur Radiol 23:2996-3004

16. Vaughan JT, Garwood M, Collins CM et al (2001) 7T vs. 4T: RF power, homogeneity, and signal-to-noise comparison in head images. Magn Reson Med 46:24-30

17. Teeuwisse WM, Brink WM, Webb AG (2012) Quantitative assessment of the effects of high-permittivity pads in 7 Tesla MRI of the brain. Magn Reson Med 67:1285-1293

18. Busse RF, Brau AC, Vu A et al (2008) Effects of refocusing flip angle modulation and view ordering in $3 \mathrm{D}$ fast spin echo. Magn Reson Med 60:640-649

19. van der Kolk AG, Zwanenburg JJ, Brundel M et al (2015) Distribution and natural course of intracranial vessel wall lesions in patients with ischemic stroke or TIA at 7.0 Tesla MRI. Eur Radiol 25:1692-1700

20. Swartz RH, Bhuta SS, Farb RI et al (2009) Intracranial arterial wall imaging using high-resolution 3-tesla contrast-enhanced MRI. Neurology 72:627-634

21. Dieleman N, van der Kolk AG, Zwanenburg JJ et al (2015) Relations between location and type of intracranial atherosclerosis and parenchymal damage. J Cereb Blood Flow Metab. doi:10.1177 /0271678X15616401

22. Klein S, Staring M, Murphy K, Viergever MA, Pluim JP (2010) elastix: a toolbox for intensity-based medical image registration. IEEE Trans Med Imaging 29:196-205

23. Kuijf HJ, van Veluw SJ, Viergever MA, Vincken KL, Biessels GJ (2013) How to assess the reliability of cerebral microbleed rating? Front Aging Neurosci 5:57

24. Versluis MJ, Peeters JM, van Rooden S et al (2010) Origin and reduction of motion and $\mathrm{f} 0$ artifacts in high resolution $\mathrm{T} 2 *$-weighted magnetic resonance imaging: application in Alzheimer's disease patients. Neuroimage 51:1082-1088

25. van der Kolk AG, Hendrikse J, Luijten PR (2012) Ultrahigh-field magnetic resonance imaging: the clinical potential for anatomy, pathogenesis, diagnosis, and treatment planning in brain disease. Neuroimaging Clin N Am 22:xii

26. Xie Y, Yang Q, Xie G, Pang J, Fan Z, Li D (2015) Improved blackblood imaging using DANTE-SPACE for simultaneous carotid and intracranial vessel wall evaluation. Magn Reson Med 75:22862294

27. Ryoo S, Cha J, Kim SJ et al (2014) High-resolution magnetic resonance wall imaging findings of Moyamoya disease. Stroke 45: $2457-2460$

28. van der Kolk AG, Zwanenburg JJ, Denswil NP et al (2015) Imaging the intracranial atherosclerotic vessel wall using 7T MRI: initial comparison with histopathology. AJNR Am J Neuroradiol 36: 694-701

29. Lopez-Cancio E, Dorado L, Millan M et al (2012) The BarcelonaAsymptomatic Intracranial Atherosclerosis (AsIA) study: prevalence and risk factors. Atherosclerosis 221:221-225

30. Shen Y, Goerner FL, Snyder C et al (2015) T1 relaxivities of gadolinium-based magnetic resonance contrast agents in human whole blood at 1.5, 3, and 7 T. Invest Radiol 50:330-338

31. Skarpathiotakis M, Mandell DM, Swartz RH, Tomlinson G, Mikulis DJ (2013) Intracranial atherosclerotic plaque enhancement in patients with ischemic stroke. AJNR Am J Neuroradiol 34:299304

32. Vakil P, Vranic J, Hurley MC et al (2013) T1 gadolinium enhancement of intracranial atherosclerotic plaques associated with symptomatic ischemic presentations. AJNR Am J Neuroradiol 34:2252 2258

33. Qiao Y, Zeiler SR, Mirbagheri S et al (2014) Intracranial plaque enhancement in patients with cerebrovascular events on highspatial-resolution MR images. Radiology 271:534-542

34. Portanova A, Hakakian N, Mikulis DJ, Virmani R, Abdalla WM, Wasserman BA (2013) Intracranial vasa vasorum: insights and implications for imaging. Radiology 267:667-679

35. Lorenz MW, Markus HS, Bots ML, Rosvall M, Sitzer M (2007) Prediction of clinical cardiovascular events with carotid intimamedia thickness: a systematic review and meta-analysis. Circulation 115:459-467

36. Kleinloog R, Korkmaz E, Zwanenburg JJ et al (2014) Visualization of the aneurysm wall: a 7.0-tesla magnetic resonance imaging study. Neurosurgery 75:614-622, discussion 622

37. Viessmann O, Li L, Benjamin P, Jezzard P (2016) T2-Weighted intracranial vessel wall imaging at 7 Tesla using a DANTEprepared variable flip angle turbo spin echo readout (DANTESPACE). Magn Reson Med. doi:10.1002/mrm.26152

38. Zhu C, Haraldsson H, Tian B et al (2016) High resolution imaging of the intracranial vessel wall at 3 and $7 \mathrm{~T}$ using 3D fast spin echo MRI. MAGMA 29:559-570

39. Madai VI, von Samson-Himmelstjerna FC, Sandow N et al (2015) Ultrahigh-field MPRAGE Magnetic Resonance Angiography at 7.0T in patients with cerebrovascular disease. Eur J Radiol 84: 2613-2617 